\title{
MANIFESTACIONES CLIINICAS Y RESULTADOS MATERNO-PERINATALES DEL COVID-19 ASOCIADO AL EMBARAZO: UNA REVISIÓN SISTEMÁTICA
}

\author{
CLINICAL MANIFESTATIONS AND MATERNAL-PERINATAL RESULTS OF COVID-19 ASSOCIATED WITH \\ PREGNANCY: A SYSTEMATIC REVIEW
}

\author{
Marco Antonio Chilipio-Chiclla (iD) 1,a, Karen Elizabeth Campos-Correa (iD) 2,a
}

\begin{abstract}
Objetivo: Evaluar las manifestaciones clínicas y resultados materno-perinatales de la infección por COVID-19 asociada al embarazo. Material y métodos: Se revisaron ocho bases de datos electrónicas (Science Direct, Pubmed, Lilacs, Scielo, Redalyc, LitCovid, EBSCO y Scopus). Se consideraron estudios primarios a texto completo en idioma español e inglés; publicados entre el 01 de enero y 15 de mayo de 2020; y realizados en gestantes con diagnóstico confirmado de COVID-19 (reacción en cadena polimerasa con transcripción inversa [RT-PCR]. Resultados: Se incluyeron 15 estudios que informaron 224 casos de infección por COVID-19 asociada al embarazo, principalmente en gestantes del tercer trimestre (13/15). La tos y fiebre fueron las principales manifestaciones clínicas reportadas en todas las investigaciones. Menos de la tercera parte de estudios muestran al dolor abdominal como manifestación obstétrica que motiva la búsqueda de atención. El antecedente epidemiológico de exposición y las alteraciones en exámenes auxiliares de laboratorio (linfopenia y PCR elevado, principalmente) son reportadas con regular frecuencia. La enfermedad por COVID-19 asociado al embarazo se presenta principalmente de forma leve; asimismo, la mortalidad e ingreso a UCI son resultados maternos exclusivamente asociados a las pocas presentaciones severas. La cesárea es la vía de culminación mayormente empleada, siendo en pocos casos la neumonía por COVID-19 la indicación citada. El bajo peso al nacer y el ingreso a UCIN son resultados perinatales descritos en 9 de 15 estudios, existiendo poca mortalidad perinatal. Solo en tres estudios se reporta que existe transmisión vertical. Conclusión: La enfermedad por COVID-19 asociado al embarazo se reporta principalmente en gestantes de tercer trimestre, con fiebre y tos presentes a la admisión, siendo mayormente casos leves. Asimismo, genera pocas complicaciones materno-perinatales; sin embargo, en casos severos la morbimortalidad es más frecuente.
\end{abstract}

Palabras clave: Evaluación de síntomas, Complicaciones del embarazo, Infecciones por coronavirus, Resultado del embarazo (Fuente: DeCS, BIREME)

Objective: To evaluate the clinical manifestations and maternal-perinatal results of COVID-19 infection associated with pregnancy. Material and methods: Eight electronic databases (Science Direct, Pubmed, Lilacs, Scielo, Redalyc, LitCovid, EBSCO and Scopus) were reviewed. Full text primary studies in Spanish and English were considered; published between January 1 and May 15, 2020; and performed in pregnant women with a confirmed diagnosis of COVID-19 (reverse transcription polymerase chain reaction [RT-PCR]. Results: 15 studies were included that reported 224 cases of pregnancy-associated COVID-19 infection, mainly in third trimester pregnant women (13/15). Cough and fever were the main clinical manifestations reported in all the investigations. Less than a third of studies show abdominal pain as an obstetric manifestation that motivates attention seeking. The epidemiological history of exposure and the alterations in auxiliary laboratory tests (lymphopenia and high CRP, mainly) are reported regularly. COVID-19 disease associated with pregnancy occurs mainly mildly; Likewise, mortality and ICU admission are maternal outcomes exclusively associated with the few severe presentations. Cesarean section is the most used route of completion, with COVID-19 pneumonia being the cited indication in a few cases. Low birth weight and NICU admission are perinatal outcomes described in 9 of 15 studies, with little perinatal mortality. Only in three studies is vertical transmission reported. Conclusion: COVID-19 disease associated with pregnancy is mainly reported in third trimester pregnant women, with fever and cough present at admission, being mostly mild cases. Likewise, it generates few maternal-perinatal complications; however, in severe cases, morbidity and mortality becomes more frequent.

Key words: Symptom assessment, Pregnancy complications, Coronavirus infections, Pregnancy outcome (Source: MeSH NLM)

Filiación:

1. Licenciado en Obstetricia, Maestría en Bioestadística

2. Licenciada en Obstetricia, Maestría en Epidemiología Clínica

a. Institute of Development Research for Professionals. Lima, Perú.
Citar como: Chilipio-Chiclla MA, Campos-Correa KE. Manifestaciones clínicas y resultados maternoperinatales del COVID-19 asociado al embarazo: Una revisión sistemática. Revista Internacional de Salud Materno Fetal. 2020; 5 (2): 24-37.
Financiamiento: Este artículo ha sido financiado enteramente por los autores. Conflictos de interés: Los autores no declaran conflicto de interés alguno. Correspondencia: Marco Antonio Chilipio Chiclla (marco.chilipio@unmsm.edu.pe)

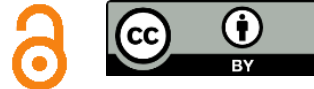

Recibido: 21 de Junio del 2020 Aprobado: 03 de Julio del 2020 Publicado: 03 de Julio del 2020 


\section{INTRODUCCIÓN}

A casi dos meses desde que la Organización Mundial de la Salud (OMS) declarase pandemia a la enfermedad del COVID-19, ya bordean los 5 millones de casos confirmados y un tercio de millón de muertes a nivel mundial (1). El incremento de casos se ha acompañado de una rápida generación de evidencias científicas de diversa índole; entre ellas, aquellas que caracterizan el curso clínico y resultados de la enfermedad $(2,3)$. Al día de hoy publicaciones ya esbozan la existencia de ciertos grupos poblacionales vulnerables, entre los cuales destacan aquellos de edades avanzadas(4). Análogamente, la fisiología del embarazo caracterizada por periodos pro y antiinflamatorios de adaptación del aloinjerto fetal (5) hace suponer que las gestantes son un grupo propenso a infecciones virales $(6,7)$, entre ellas el SARS-CoV-2, según sugieren recientes publicaciones $(8,9)$.

Epidemias anteriores por coronavirus humanos como el SARS-CoV-1 y el MERS-CoV, causantes de graves estragos sanitarios en 2003 y 2012, respectivamente, sugieren que las gestantes son un grupo de riesgo que generó alta incidencia de complicaciones maternas y perinatales $(10,11)$, e incluso mortalidad (12). Al día de hoy, aún es prematuro afirmar si el COVID-19 asociado al embarazo genera mayor o menor impacto en los resultados maternos y perinatales, pues la generación de evidencias científicas en población obstétrica todavía sigue su curso. No obstante, es necesario consolidar lo que hasta el momento se conoce del COVID-19 en población obstétrica. Yuen et al. (13) afirman que solo los esfuerzos de investigación sobre COVID-19 "...solidificarán la base científica sobre la cual se toman decisiones importantes". En el marco de estas precisiones, el propósito de este manuscrito es resumir sistemáticamente la literatura existente sobre las manifestaciones clínicas y resultados materno-perinatales asociados a la infección por COVID-19 en población obstétrica.

\section{MATERIAL Y MÉTODOS}

Se emplearon las directrices de la declaración PRISMA (Preferred Reporting Items for Systematic reviews and Meta-Analyses) (14) para revisiones sistemáticas. De la estrategia PICO se excluyó el comparador (C) pues las evidencias científicas disponibles (al 15 de mayo de 2020) sobre COVID-19 en población obstétrica consistían en reportes y series de caso. Se definió como población $(P)$ a gestantes; como intervención (I) a la enfermedad por COVID-19; y como resultados $(\mathrm{O})$ a las manifestaciones clínicas y resultados materno-perinatales. El ingreso de revisión sistemática al registro PROSPERO (International Prospective Register of Systematic Reviews) se omitió, dada la urgente necesidad de evidencias científicas para apoyar la toma de decisiones.

\section{CRITERIOS DE ELEGIBILIDAD}

Se seleccionaron publicaciones científicas que tuvieron población obstétrica con diagnóstico de COVID-19, de las cuales se describan manifestaciones clínicas y resultados maternoperinatales. Se incluyeron también publicaciones con neonatos como población, de los cuales fue posible recuperar datos maternos. Se consideraron investigaciones primarias en idioma inglés o español publicadas entre el 1 de enero y 15 de mayo de 2020 .

\section{FUENTES DE INFORMACIÓN}

Se realizó una revisión en bases de datos de acceso libre (Science Direct, Medline [vía Pubmed], Lilacs, Scielo y Redalyc) y por suscripción (Scopus y EBSCO); asimismo, no se estableció contacto con los autores de las publicaciones.

\section{BÚSQUEDA}

La terminología de búsqueda empleada fueron términos MESH (Medical Subject Headings) y DeCS (Descriptores en Ciencias de la Salud), disponibles en http://www.ncbi.nlm.nih.gov/mesh y http://decs.bvs.br, respectivamente.

Los descriptores seleccionados para cada componente de la estrategia inicial fueron: 1) para Población (P): "Pregnancy" y "Pregnancy, High risk"; 2) para Intervención (I): "Coronavirus Infections", "COVID-19" y "SARS-CoV-2"; y 3) para Resultados (O): "Pregnancy Complications", "Pregnancy Complications, Infectious", "Pregnancy outcome", "Mortality", "Maternal Mortality", "Maternal Death", "Perinatal Death", "Infant, Newborn", "Fetal Death", "Stillbirth" y "Perinatal Mortality".

El algoritmo de búsqueda empleado fue: \#1 AND \#2 AND \#3. Dicho algoritmo fue usado en todas las 
bases de datos con excepción de Science Direct, donde fue necesario bifraccionar el componente Resultados (O) pues dicho buscador solo admitió hasta ocho operadores booleanos.

\section{SELECCIÓN DE ESTUDIOS}

Mediante los filtros metodológicos disponibles en las bases de datos consultadas, se seleccionaron: i) investigaciones publicadas hasta el 15 de mayo del presente año; ii) estudios con población obstétrica con COVID-19 confirmado por test de reacción en cadena de la polimerasa de transcripción inversa (RT-PCR); y iii) estudios a texto completo. Se excluyeron estudios con población obstétrica sin diagnóstico confirmado de COVID-19

\section{PROCESO DE EXTRACCIÓN DE DATOS}

La recuperación inicial de artículos fue realizada por M.A.C.C., así como la verificación de estudios elegibles por título y resumen. En la revisión a texto completo y extracción de datos participaron la coautora K.E.C.C., quien participó de la revisión independiente de artículos. Asimismo, toda discrepancia fue resuelta por consenso.

\section{LISTA DE DATOS}

Las características maternas y manifestaciones clínicas analizadas fueron: edad, trimestre gestacional a la admisión, comorbilidad, sintomatología a la admisión, antecedente epidemiológico, evaluación auxiliar hematológica, bioquímica y tomográfica. Los resultados maternos estudiados fueron complicación obstétrica, ingreso a unidad de cuidados intensivos (UCI), mortalidad materna, resultado de la admisión, parto prematuro, parto por cesárea e indicación. Los resultados perinatales estudiados fueron: edad y peso al nacer promedios al nacimiento, bajo peso al nacer, sufrimiento fetal agudo (SFA), mortalidad perinatal (fetal y neonatal), asfixia neonatal, Apgar bajo $(<7)$, ingreso a UCI neonatal y transmisión vertical.

\section{RIESGO DE SESGO}

Se empleó una herramienta para evaluar la calidad y riesgo de sesgo propuesta por Murad et al. (15) diseñada para reportes y series de caso, y adaptada de otras ampliamente utilizadas (16). Cuenta con ocho criterios de valoración comprendidos en cuatro dominios: selección, verificación, causalidad y reporte.

\section{MEDIDAS DE RESUMEN}

Las variables cuantitativas se expresaron como medias (desviación estándar [DE]) o medianas (rango intercuartílico [RIQ]). Asimismo, datos categóricos fueron resumidos con frecuencias absolutas y relativas.

\section{RESULTADOS}

\section{SELECCIÓN Y CARACTERÍSTICAS DE LOS ESTUDIOS}

Se identificaron inicialmente 270 artículos procedentes de las ocho bases de datos consultadas. Se eliminaron 109 registros duplicados y quedaron 162 para evaluación por título y resumen. Fueron elegibles solo 23 artículos, todos evaluados a texto completo y se consideró no elegibles 8 publicaciones (Figura 1). Finalmente, se incluyeron 15 artículos en la presente revisión, siendo la mayor parte (10/15) de procedencia China: 7 estudios realizados en hospitales de la ciudad de Wuhan (provincia de Hubei) $(17,18,19,20,21,22,23) ; 2$ estudios, en provincias distintas al epicentro de la pandemia del COVID-19 $(24,25)$; y 1 , que reporta casos procedentes de 25 hospitales de diferentes provincias chinas (26). La menor parte de artículos incluidos en esta revisión (05/10) corresponden a estudios de otros países del Asia (01) (27), Europa (01), (28) Norteamérica (02) $(29,30)$ y Latinoamérica (01) (31). Es importante destacar que la única publicación latinoamericana hasta la fecha en que se delimitó la revisión y bajo los criterios de selección ya establecidos fue un artículo peruano (31) que reporta un caso severo de COVID19 asociado al embarazo (Figura 1).

El tipo de evidencias científicas disponibles hasta la fecha en que se delimitó esta revisión correspondían a series y reportes de caso en población obstétrica. Por eso, el riesgo de sesgo de las 15 publicaciones científicas incluidas se evaluó según la metodología propuesta por Murad et al. (15), que cuenta con 8 indicadores y está diseñada precisamente para este tipo de estudios. Esta herramienta es una adaptación de la escala de Newcastle-Ottawa (16), de la cual elimina elementos relacionados a comparabilidad y ajuste (aspectos no 


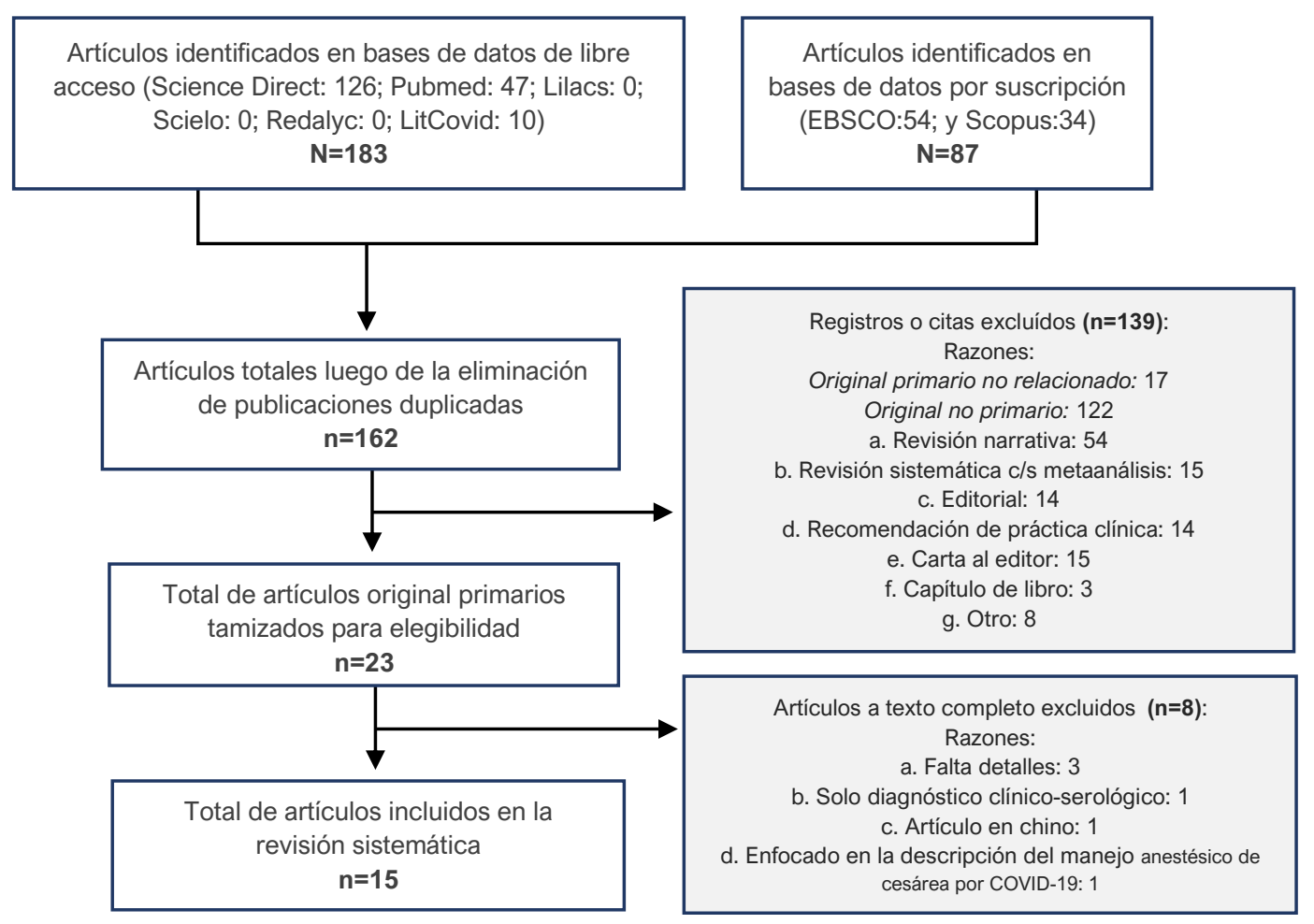

Figura 1. Diagrama de flujo del proceso de selección de artículos

relevantes en estudio no comparativos) para mantener elementos centrados en la selección, representatividad, verificación y exposición. El 66.6\% (10) de estudios incluidos cumplieron favorablemente todos los ítems aplicables y $33.4 \%$ cumplieron todos los aplicables con excepción de uno, suficientes para catalogarlos como estudios con riesgo de sesgo bajo (Tabla 1).

\section{MANIFESTACIONES CLÍNICAS}

El COVID-19 asociado al embarazo se reporta en mujeres con edades que oscilan entre 20 y 42 años. Se ubican predominantemente en el III trimestre de embarazo, seguido de aquellas de II trimestre. En menor proporción son gestantes de I trimestre y solo un estudio reporta (29) readmisión en el puerperio mediato por sintomatología COVID-19. En 6 de 15 estudios se reporta la comorbilidad materna, siendo el asma de mayor frecuencia (18.6\%)(29), seguida de obesidad (29,27), diabetes mellitus (29,31) e hipotiroidismo $(22,18)$; en menor proporción, se reportan otras como hipertensión arterial crónica (29), anemia (18) y hepatitis B (24) (Tabla 2a).
La fiebre y tos son las principales manifestaciones clínicas del COVID-19 asociadas al embarazo, de las cuales dos terceras partes de investigaciones centran su interés en describir la presencia de fiebre tanto en admisión como en el postparto $(26,25,17,18,19,21)$. La fiebre al ingreso es reportada con frecuencias entre $15.4 \%$ (25) y $86.6 \%$ (17) de casos. La fiebre postparto y tos se describen con frecuencias de hasta $67 \%$ (19) y $60 \%$ (17), respectivamente; no obstante, pueden estar presentes en el $100 \%$ de casos si existe manifestación severa de la enfermedad (27). Cuanto menos, la disnea está presente en el $6.6 \%$ de casos en COVID-19 leve (17); no obstante, está presente en el $66.6 \%$ cuando el COVID-19 es severo (27). La mialgia, malestar y fatiga son síntomas que se superponen en gran medida, siendo así Breslin et al. (29) reportan la presencia conjunta de mialgia-fatiga en el $25.5 \%$ de mujeres COVID-19 positivas (Tabla 2a).

Entre los síntomas poco frecuentes se describe la diarrea en 1.5\% (26) hasta 14.2\% (22) de admisiones por COVID-19. El dolor de garganta está presente entre el $6.6 \%$ (17) hasta el 22.2\% (19). El dolor en el pecho está presente hasta en el 11.6\% (29) de casos. 


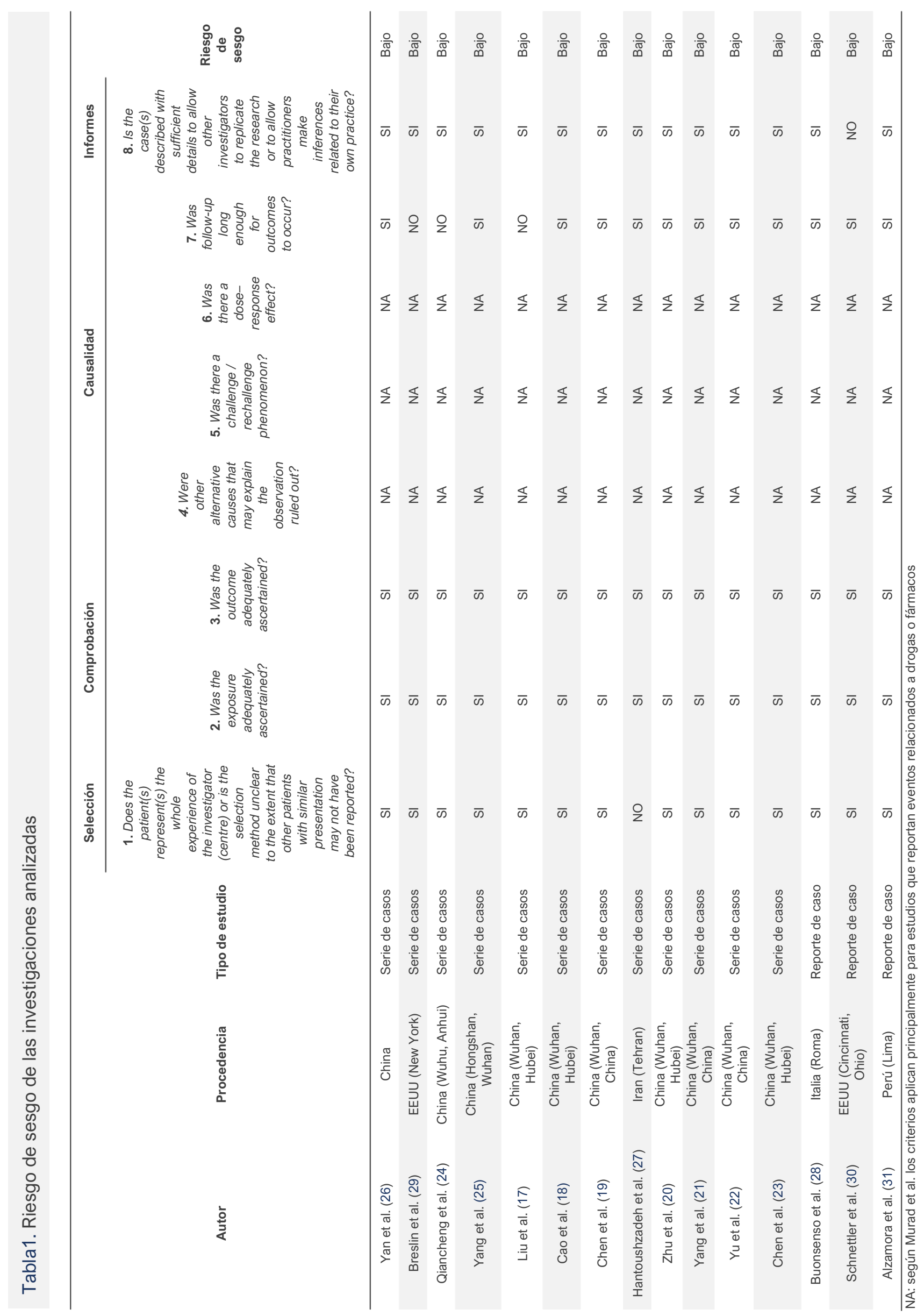




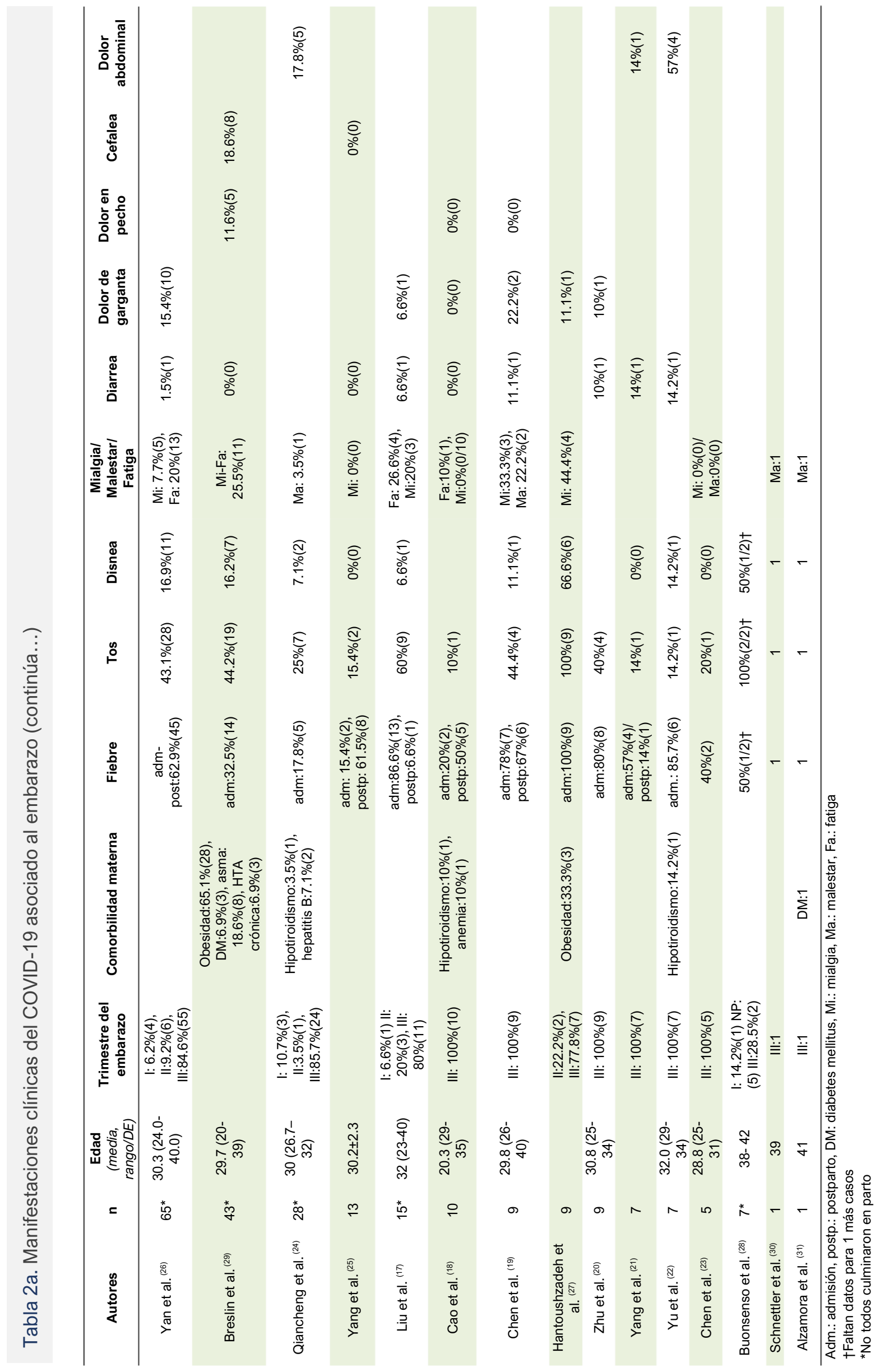




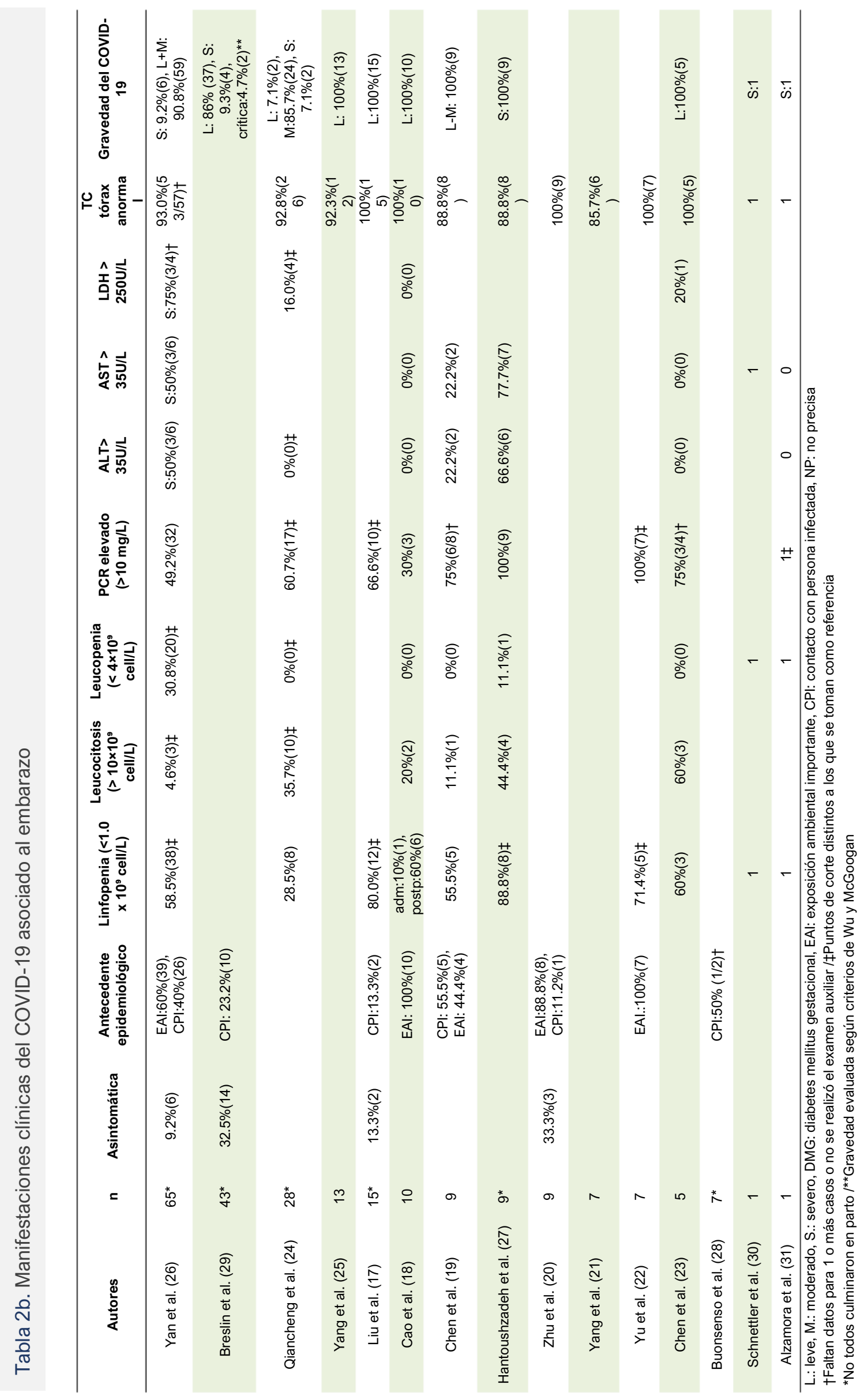


Asimismo, llegan a alcanzar frecuencias del $22.2 \%$ y, respectivamente. La cefalea solo es descrita en 2 investigaciones, la primera para negar su presencia (24) y la segunda para mostrar una frecuencia de $16.8 \%$ (29). Solo tres estudios $(24,21,22)$ reportan la presencia de síntomas obstétricos como el dolor abdominal que motiva el ingreso por emergencia. En 4 investigaciones reportan casos asintomáticos en la admisión, con frecuencia que alcanzan hasta el $33.3 \%$. En 9 estudios se reporta al antecedente epidemiológico COVID-19, siendo la exposición ambiental importante (EAI) más prevalente que el contacto con persona infectada (CPI) (Tabla 2a).

Las linfopenia y proteína C reactiva (PCR) incrementada son alteraciones que se muestran con mayor frecuencia en los estudios (10 de 15) con frecuencias máximas de $80.0 \%$ (17) y $100 \%$ (22), respectivamente. Solo para el caso de la linfopenia su frecuencia puede elevarse hasta $88.8 \%$ cuando se trata de COVID-19 severo (27). Los estudios (6 de 15) reportan con regular frecuencia a la leucocitosis desde $4.6 \%$ hasta $60 \%(26,23)$. Si bien la leucopenia es referida en mayores investigaciones (8 de 15), en algunas solo es para negar su presencia $(24,18,23)$ y en otras para mostrar frecuencia hasta de $30.8 \%$ (26). La elevación de enzimas hepáticas como la ALT, AST y LDH se reportan con frecuencias de aproximadamente $20 \%$, pero puede incrementarse hasta $75 \%-77.7 \%$ cuando se tratan de casos severos de COVID-19 (26,27). Casi todas las investigaciones (13 de 15) reportan haberse realizado evaluaciones tomográficas de tórax, con frecuencia desde $88.8 \%$ $(19,27)$ hasta $100 \%(25,18,20,22,23)$. El COVID-19 asociado al embarazo mayormente es leve en 5 de 11 estudios que reportan grado de enfermedad $(29,25,17,18,23)$, solo una investigación reporta predominancia de casos moderados (24) y en dos estudios se reporta la superioridad conjunta de casos leves-moderados $(26,19)$. La frecuencia de COVID-19 severo asociado al embarazo alcanza el $9.2 \%$ (26) y Breslin et al. (29) reportan casos críticos en $4.7 \%$ de admisiones (Tabla $\mathbf{2 b}$ ).

\section{RESULTADOS MATERNOS Y PERINATALES}

Las complicaciones obstétricas observadas en 10 de 15 estudios, siendo los trastornos hipertensivos, rotura prematura de membranas (RPM) y diabetes mellitus gestacional (DMG) los más reportados. En menor frecuencia estudios muestran a la placenta previa. El ingreso a unidad de cuidados intensivos $(\mathrm{UCl})$ se reporta solo en tres investigaciones, con frecuencias entre $4.6 \%$ a $9.2 \%(29,26)$. Solo una investigación muestra reportes de mortalidad materna, todas procedentes de casos COVID-19 severo (27). En todos estudios analizados el parto fue el principal resultado de la atención, seguido de la continuación del embarazo y en menor porcentaje se cita a la interrupción del embarazo por aborto $(26,24,28)$. Solo en una investigación se reportan dos casos de interrupción del embarazo por mortalidad materna-fetal (27) y en otra, una readmisión puerperal por sintomatología COVID-19 (29) (Tabla 3).

La cesárea es la principal vía de culminación del embarazo, con frecuencias entre $40 \%$ y $100 \%$. Asimismo, en tres series $(26,19,21)$ y un reporte $(31)$ de caso se cita a la neumonía por COVID-19 como la indicación de cesárea. En cinco estudios se reportan alteraciones de la frecuencia cardiaca fetal como indicación de cesárea $(29,27,23,28,30)$, y en tres, al sufrimiento fetal agudo $(26,18,19)$. Otras indicaciones regularmente frecuentes de cesárea son la preeclampsia, cesárea previa, trabajo de parto disfuncional e hipertransaminasemia; en menor frecuencia, se citan otras indicaciones como inducción fallida, embarazo gemelar, edad materna añosa y antecedente de óbito fetal (Tabla 3). La mayor frecuencia reportada de bajo peso al nacer (BPN), prematuridad y sufrimiento fetal agudo (SFA) es $77.7 \%, 66.6 \%$ y $15.9 \%$, respectivamente $(20,26)$. La mortalidad fetal solo se reporta en un estudio con $44.4 \%$ de casos, todos procedentes de madres afectadas por COVID-19 severo (27). Solo un estudio reporta asfixia neonatal en $2 \%$ de nacimientos (26) y dos investigaciones reportan un caso -cada una- de Apgar bajo al minuto $(27,31)$, ambos de embarazos con manifestaciones severas de la enfermedad. Según las investigaciones que incluyen mayor número de casos, la frecuencia de ingreso a Unidad de Cuidados Intensivos Neonatales (UCIN) oscila entre $16.6 \%$ (29) hasta $34.0 \%$ (26). Asimismo, la mortalidad neonatal, con excepción de dos series de casos muy pequeñas $(27,20)$, apenas alcanza el $2 \%$ de nacimientos. Tres investigaciones confirman transmisión vertical del COVID-19, con un caso cada una $(28,31,22)$ y en otra publicación reportan un caso indeterminado (29) (Tabla 4). 


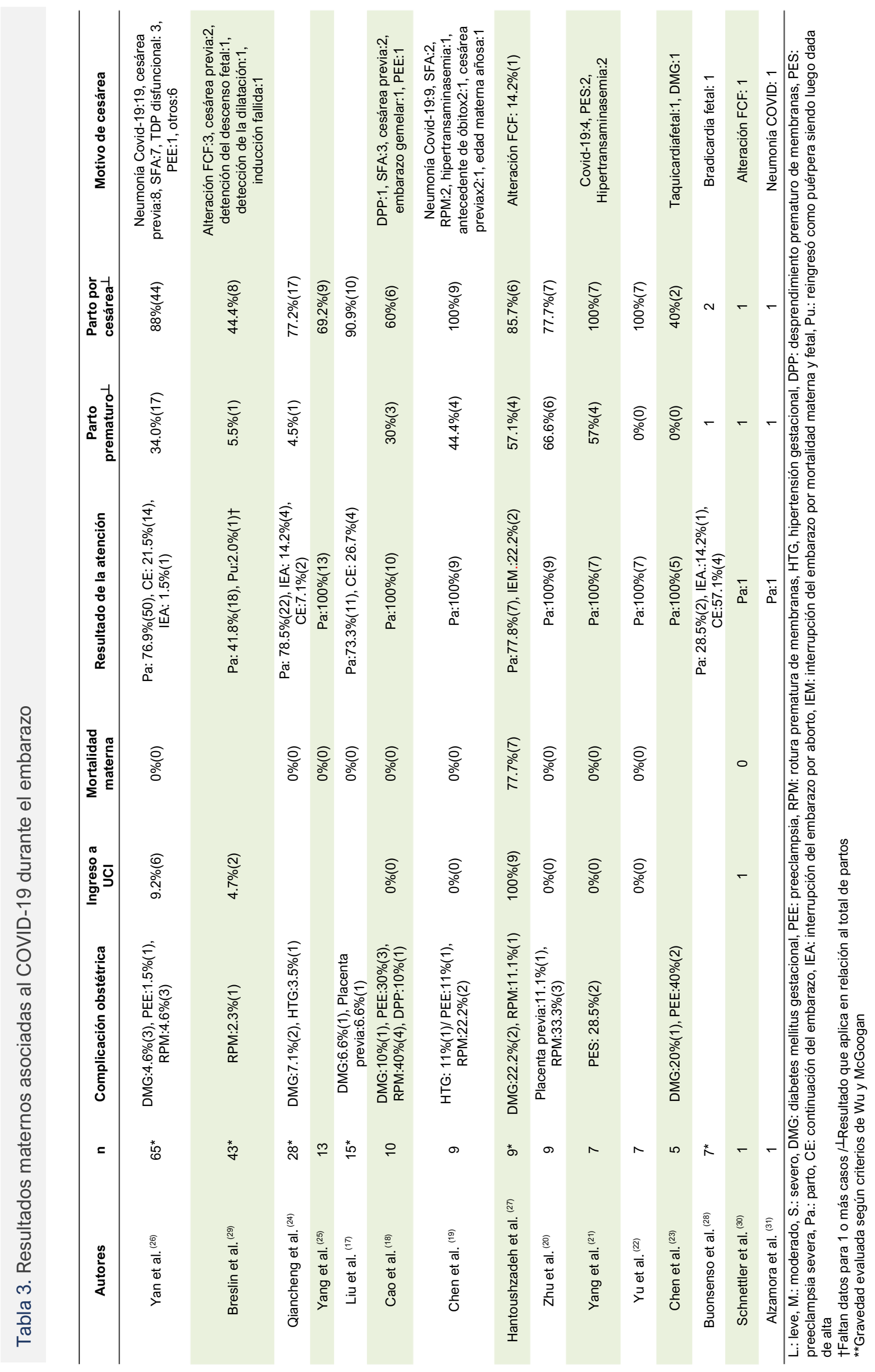




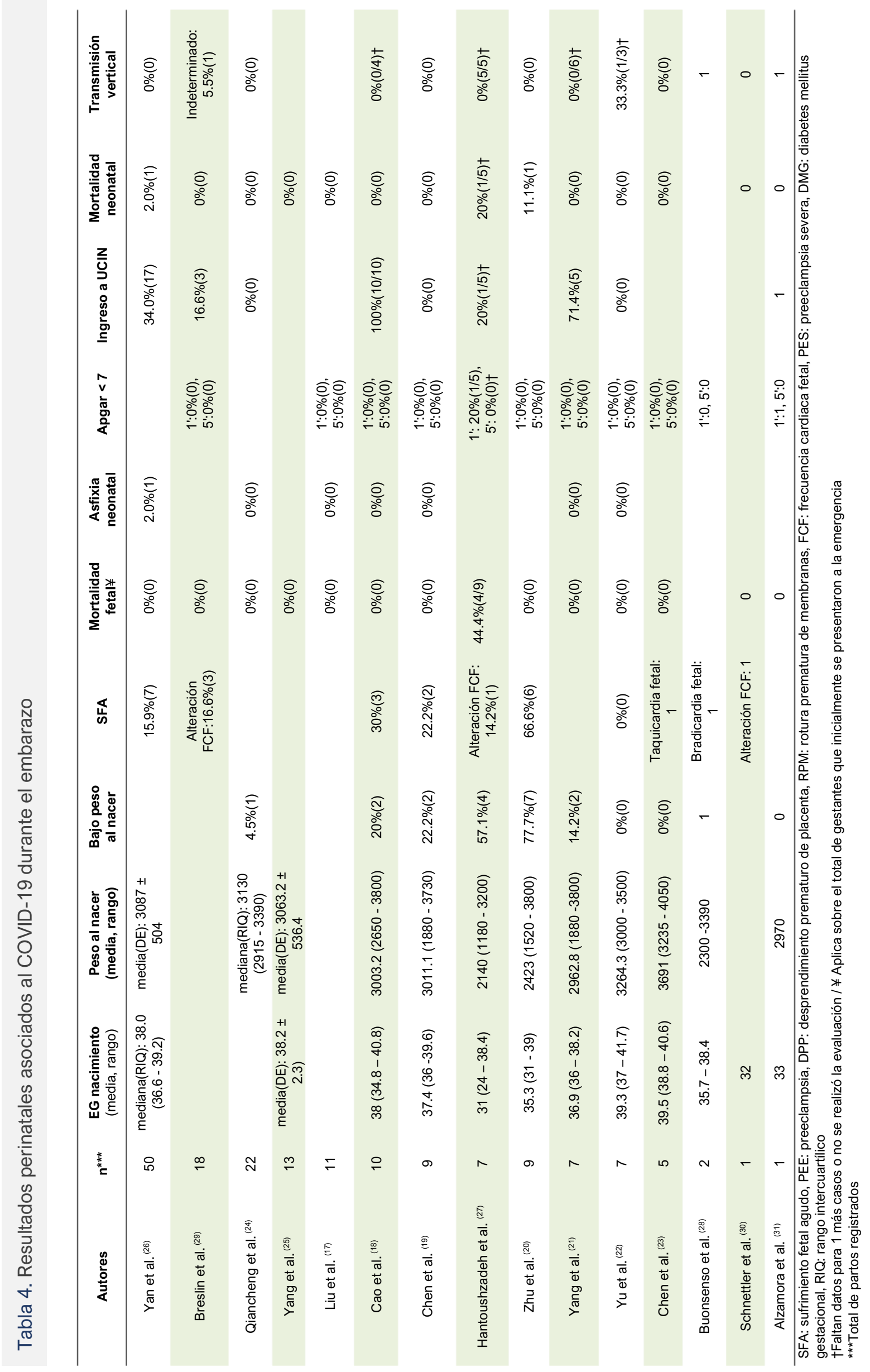




\section{DISCUSIÓN}

La pandemia por la enfermedad del coronavirus es el problema de salud global que sigue generando a junio de 2020 el colapso de diversos sistemas de salud. La generación de evidencias científicas es una necesidad imperante, particularmente para poblaciones susceptibles como las gestantes, en quienes las características y resultados de la infección por COVID-19 siguen siendo limitadas y fragmentadas. Por ello, esta revisión busca resumir sistemáticamente lo que se conoce hasta el momento.

El COVID-19 apenas cumplirá seis meses en el mundo desde que la OMS publicase su primer parte; por eso, existe alta probabilidad de que muchas gestantes reportadas hayan contraído la infección en un periodo cercano al parto. Sumado a esto, la restricción impuesta del acceso a servicios de salud para demandas de atención no COVID-19 como la atención prenatal ha limitado la identificación de muchas gestantes infectadas en etapas iniciales del embarazo y no sean reportadas con similar frecuencia con que son descritas gestantes del tercer trimestre. Probablemente estas son las razones del porqué las investigaciones analizadas reportan con mayor frecuencia gestantes del tercer trimestre. La edad de las gestantes con diagnóstico de COVID-19 usualmente se sitúa entre la segunda y tercera década de vida; y ciertamente, situarse en edades reproductivas recomendables (20-35 años) confiere menor riesgo de eventos adversos comúnmente relacionados con los extremos de la edad reproductiva (32) Sin embargo, 6 de 15 estudios analizados reportan comorbilidades maternas, lo cual confiere un riesgo agregado al que se supone genera la enfermedad del COVID-19 (33). Esto es especialmente importante para gestantes con comorbilidades como hipertensión arterial y diabetes mellitus, pues incrementan 2.0 (34) y 3.2 (35) veces el riesgo de enfermedad grave. En igual situación se encuentra la obesidad, que incluso incrementa la estadía hospitalaria, ingreso a unidades críticas y eleva la frecuencia de mortalidad $(36,37)$. Si bien estas presunciones se basan en hallazgos de estudios realizados en población general, es probable se corroboren en sucesivas investigaciones con mayor nivel de evidencia que las escasas descripciones que se disponen hasta el momento para población obstétrica.
Todas las investigaciones (15/15) muestran a la fiebre y tos como principales manifestaciones clínicas del COVID-19 asociado al embarazo, presentes hasta en un $86.6 \%$ y $60 \%$ de admisiones (17) respectivamente. Según el metaanálisis de Fu et al. (38) la fiebre $(83.3 \%)$ y tos $(60.3 \%)$ son también las características clínicas más prevalentes en población adulta en general, lo cual evidencia elevada similitud del comportamiento clínico de la enfermedad. Incluso una reciente investigación peruana (39) realizada en población adulta no gestante sitúa a fiebre $(78.6 \%)$ y tos $(71.4 \%)$ como las principales manifestaciones clínicas, lo cual refuerza dicha similitud. Dado que son evaluaciones de ínfimo costo y elevada rapidez, esto obliga a pesquisar su búsqueda en toda gestante que ingresa por emergencia. Complementariamente, conviene pesquisar el antecedente epidemiológico pues gran parte de estudios (9 de 15) reportan su presencia. Son 5 los estudios -todos de China- que reportan la exposición ambiental importante (EAl), refiriéndose a residir en zonas contiguas y/o haber viajado en el último mes a la ciudad de Wuhan (Hubei); sin embargo, para la etapa en que se ubica el desarrollo de la pandemia, basta con indagar el antecedente de contacto con una persona COVID-19 positiva.

Existe elevado interés en describir la presencia de fiebre tanto al ingreso como durante la estancia hospitalaria, periodo en donde llega a alcanzar frecuencias de $67 \%$ (19). Esto obliga a adoptar una actitud siempre expectante ante este signo; especialmente, si se considera que un $32.5 \%$ (29)$33.3 \%$ (20) de mujeres ingresan asintomáticas. En la evaluación auxiliar, la linfopenia y el PCR elevado son hallazgos laboratoriales más citados en 10 de 15 estudios analizados, pudiendo alcanzar frecuencias del $80 \%$ y $75 \%$, respectivamente. Estas cifras coindicen con los resultados del metaanálisis de Fang et al. (40) donde también son los hallazgos laboratoriales más frecuentes para población adulta general. No obstante, se requieren series de caso con poblaciones obstétricas más grandes para corroborarlo.

La tomografía torácica es la evaluación con mayor sensibilidad para detectar la presencia del COVID-19 asociada al embarazo, en 5 de 13 reportes que muestran su realización, su alteración alcanza frecuencias entre $88.8 \%-100 \%(17,18,20,22,23)$. Esto es consistente con el estudio de Ai et al. (41) donde 
se le atribuye una sensibilidad del $97 \%$. Si bien Sun et al. (42) añaden que carece de especificidad suficiente para distinguirla de otras neumonías no COVID-19, conviene siempre tomar con cautela toda anormalidad y correlacionarla con la clínica. La gravedad de la enfermedad por el nuevo coronavirus asociada al embarazo es predominantemente leve, lo cual concuerda con el estudio de Chen et al. (43) realizado en población general. No obstante, en mínima proporción se dan casos severos con frecuencias de $7.1 \%$ (26) y $9.3 \%$ (29), lo cual varía según los criterios empleados para definir la gravedad.

Las complicaciones obstétricas son regularmente frecuentes en el COVID-19 asociado al embarazo, destacando la preeclampsia, diabetes mellitus gestacional y rotura prematura de membranas. Aparentemente, estas complicaciones suceden con similar frecuencia a embarazos no afectados por el nuevo coronavirus (44). Aún es prematuro afirmar que la infección por el nuevo coronavirus no incrementa el riesgo de dichas complicaciones, ya que mayoritariamente $(\approx 80 \%)$ las gestantes reportadas en las investigaciones se encuentran en el tercer trimestre. Esto limitaría la exposición del embarazo al virus, dejando escaso espacio para que la fisiopatología progrese hacia dichas complicaciones. El ingreso a UCI tiene una frecuencia del 9.2\% (26) y se relaciona estrechamente con el COVID-19 severo, tal como lo muestra un estudio iraní (27). La mortalidad materna es otro resultado estrictamente relacionado con la enfermedad severa (27). El parto por cesárea se reporta en todas las investigaciones, aunque con magnitud variable; no obstante, solo en tres estudios se cita la neumonía por COVID-19 como su indicación $(26,19,21)$. La neumonía no es citada como indicación de cesárea en consensos para la práctica clínica (45), pero es altamente probable que el trastorno ventilatorio reduzca el flujo uteroplacentario y se instalen alteraciones de la frecuencia cardiaca fetal $(30,28,23,27)$ que progresarán a sufrimiento fetal agudo $(26,18,19)$ requiriéndose de cesárea.

Las series de caso más grandes reportan frecuencias de bajo peso al nacer (BPN) (24) y asfixia neonatal (26) del $4.5 \%$ y $2.0 \%$, respectivamente. El ingreso a $\mathrm{UCl}$ neonatal se da en $34.0 \%$ de casos, según una serie que reporta 50 nacimientos (26), lo cual tendría relación directa con la prematuridad, hipoxia fetal y BPN; e indirecta, con el COVID-19. La mortalidad intraútero solo se reporta en una investigación (27), asociada siempre a COVID-19 severo; mientras que la muerte neonatal se reporta en tres estudios con un caso cada uno. Todo apunta a la existencia de una relación entre manifestaciones clínicas maternas COVID-19 y resultados maternoperinatales adversos; asimismo, esta enfermedad mediaría la aparición de condiciones mórbidas, que en última instancia son responsables directas de los resultados materno-perinatales adversos.

Es importante reconocer como limitación la existencia de pocas series de caso con poblaciones suficientemente grandes como para realizar estimaciones adecuadas. Esto podría conferir algún grado de sesgo a las estimaciones proporcionadas; no obstante, es seguro que esto se resolverá en el corto y mediano plazo pues proyecciones indican que, a diciembre del 2020, habrá solo en Estados Unidos hasta 3308 casos graves de COVID-19 en población obstétrica (46), los suficientes para generar estimaciones precisas. Debido a las adaptaciones fisiológicas propias del embarazo, las mujeres embarazadas y sus fetos representan una población de alto riesgo (33); sin embargo, estudios reconocen que aún no existe evidencia suficiente para afirmar que el embarazo es un factor de riesgo para una enfermedad más grave en mujeres con COVID19(47,48).

Es posible concluir, en base a los estudios analizados, que la población obstétrica con COVID-19 se caracteriza por situarse en edades entre 20 y 42 años, cursan el tercer trimestre gestacional y presentan tos y fiebre en la admisión; asimismo, en la evaluación auxiliar muestran linfopenia, PCR elevado y anormalidad tomográfica. Mayormente son casos leves, con baja frecuencia presentan complicación obstétrica, ingreso a $\mathrm{UCl}$ y mortalidad; sin embargo, frecuentemente culminan en cesárea. Los resultados perinatales adversos son más frecuentes en mujeres con enfermedad severa por COVID-19, aunque con escaza mortalidad neonatal; finalmente, la transmisión vertical aún es incierta, por los diferentes criterios de evaluación aplicados. 


\section{REFERENCIAS}

1. Organización Mundial de la Salud. WHO Coronavirus Disease (COVID-19) Dashboard. [Online].; 2020 [cited 2020 abril 2020. [Link]

2. Huang $\mathrm{C}$, Wang $\mathrm{Y}$, Li W, Ren L, Zhao J, $\mathrm{Hu}$ L, et al. Clinical features of patients infected with 2019 novel coronavirus in Wuhan, China. Lancet. 2020; 395: 497$506 . \quad$ doi: 10.1016/S01406736(20)30183-5 [Link]

3. Liu K, Fang Y, Deng Y, Liu W, Wang M, $\mathrm{Ma} \mathrm{J}$, et al. Clinical characteristics of novel coronavirus cases in tertiary hospitals in Hubei Province. Chin Med J (Engl). 2020; 133(9): 1025-1031. doi: 10.1097/CM9.0000000000000744. [Link]

4. Wang D, Yin Y, Hu C, Liu X, Zhang X, Zhou $S$, et al. Clinical course and outcome of 107 patients infected with the novel coronavirus, SARS-CoV-2, discharged from two hospitals in Wuhan, China. Crit Care. 2020; 24: 188. doi: 10.1186/s13054-020-02895-6. [Link]

5. Prabhudas M, Bonney E, Caron E, Dey S, Erlebacher A, Fazleabas A, et al. Immune mechanisms at the maternal-fetal interface: perspectives and challenges. Nat Immunol. 2015; 16(4): 328-334. [Link]

6. Racicot K, Mor G. Risks associated with viral infections during pregnancy. J Clin Invest. 2017; 127(5): 1591-1599. doi: 10.1172/JCl87490. [Link]

7. Silasi M, Cárdenas I, Racicot K, Kwon J, Aldo $\mathrm{P}$, Mor G. Viral infections during pregnancy. Am J Reprod Immunol. 2015; 73(3): 199-213. doi: 10.1111/aji.12355. [Link]

8. Stumpfe $F$, Titzman A, Schneider $M$, Stelzl P, Kehl S, Fasching P, et al. SARSCoV-2 Infection in Pregnancy - a Review of the Current Literature and Possible Impact on Maternal and Neonatal Outcome. Geburtshilfe Frauenheilkd. 2020; 80(4): 380-390. doi: 10.1055/a1134-5951. [Link]

9. Zhao X, Jiang Y, Zhao Y, Xi H, Liu C, Qu $\mathrm{F}$, et al. Analysis of the susceptibility to COVID-19 in pregnancy and recommendations on potential drug screening. Eur J Clin Microbiol Infect Dis. 2020 Apr 23; doi: 10.1007/s10096-02003897-6. [Link]

10. Jeong S, Sung S, Hung J, Ahn S, Kang $\mathrm{E}$, Chang $\mathrm{Y}$, et al. MERS-CoV Infection in a Pregnant Woman in Korea. J Korean Med Sci. 2017; 32(10): 1717-1720. doi: 10.3346/jkms.2017.32.10.1717. [Link]

11. Martens M, Kalgi B. Middle Eastern Respiratory Syndrome and Pregnancy [18J]. Obstet. Gynecol. 2016; 127: S85S86.

10.1097/01.AOG.0000483787.93122.d5 [Link]

12. Wong S, Chow K, Leung T, Ng W, Ng T, Shek $\mathrm{C}$, et al. Pregnancy and perinatal outcomes of women with severe acute respiratory syndrome. Am. J. Obstet. Gynecol. 2004; 191: 292-297. doi: 10.1016/j.ajog.2003.11.019. [Link]

13. Yuen $K$, Ye Z, Fung $S$, Chang $C$, Jin D. SARS-CoV-2 and COVID-19: The most important research questions. Cell Biosci. 2020; 10: 40. doi: 10.1186/s13578-020-00404-4. [Link]

14. Urrútia G, Bonfill X. Declaración PRISMA: una propuesta para mejorar la publicación de revisiones sistemáticas y metaanálisis. Med Clin (Barc). 2010; 135(11): 507-511. doi: 10.1016/j.medcli.2010.01.015. [Link]

15. Murad M, Sultan S, Haffar S, Bazerbach F. Methodological quality and synthesis of case series and case reports. BM Evid Based Med. 2018; 23(2): 60-63. doi: 10.1136/bmjebm-2017-110853. [Link]

16. Stang A. Critical evaluation of the Newcastle-Ottawa scale for the assessment of the quality of nonrandomized studies in metaanalyses. Eur J Epidemiol. 2010; 25(9): 603-605. doi: 10.1007/s10654-0109491-z. [Link]

17. Liu D, Li L, Wu X, Zheng D, Wang J, Yang $L$, et al. Pregnancy and Perinatal Outcomes of Women With Coronavirus Disease (COVID-19) Pneumonia: A Preliminary Analysis. Am J Roetgenol. 2020 Jul; doi: 10.2214/AJR.20.23072. [Link]

18. Cao D, Yin H, Chen J, Tang F, Peng M, L $R$, et al. Clinical analysis of ten pregnant women with COVID-19 in Wuhan, China: A retrospective study. Int $\mathrm{J}$ Infect Dis. 2020; 95: 294-300. doi: 10.1016/j.jijid.2020.04.047. [Link]

19. Chen H, Guo J, Wang C, Luo F, Yu X, Zhang $W$, et al. Clinical characteristics and intrauterine vertical transmission potential of COVID-19 infection in nine pregnant women: a retrospective review of medical records. Lancet. 2020; 395(10226): 809-815. doi: 10.1016/S0140-6736(20)30360-3. [Link]

20. Zhu H, Wang L, Fang C, Peng S, Zhang L, Chang G, et al. Clinical analysis of 10 neonates born to mothers with 2019nCoV pneumonia. Transl Pediatr. 2020; 9(1): 51-60. doi: 10.21037/tp.2020.02.06. [Link]

21. Yang $P$, Wang $X$, Liu $P$, Wei $C, B e ~ H$, Zheng $\mathrm{J}$, et al. Clinical characteristics and risk assessment of newborns born to mothers with COVID-19. J Clin Virol. 2020; 127: $104356 . \quad$ doi: 10.1016/j.jcv.2020.104356 [Link]

22. Yu N, Li W, Kang Q, Xiong Z, Wang S, Lin $X$, et al. Clinical Features and Obstetric and Neonatal Outcomes of Pregnant Patients With COVID-19 in Wuhan, China: A Retrospective, Single-Centre, Descriptive Study. Lancet Infect Dis 2020; 20(5): 559-564. doi: 10.1016/S1473-3099(20)30176-6 [Link]

23. Chen S, Liao E, Cao D, Gao Y, Sun G, Shao Y. Clinical analysis of pregnant women with 2019 novel coronavirus pneumonia. J Med Virol. 2020; 01-06. doi: 10.1002/jmv.25789 [Link]

24. Qiancheng $X$, Jian $S$, Lingling $P$, Lei $H$, Xiaogan J, Weihua $\mathrm{L}$, et al. Coronavirus disease 2019 in pregnancy. Int J Infect Dis. 2020; 95: 376-383. doi: 10.1016/j.ijid.2020.04.065 [Link]

25. Yang $\mathrm{H}$, Sun $\mathrm{G}$, Tang $F$, Peng $M$, Gao $Y$, Peng $\mathrm{J}$, et al. Clinical Features and Outcomes of Pregnant Women Suspected of Coronavirus Disease 2019. J Infect. 2020 Apr 12; doi: 10.1016/j.jinf.2020.04.003 [Link]

26. Yan J, Guo J, Fan C, Juan J, Yu X, Li J, et al. Coronavirus disease 2019 (COVID19) in pregnant women: A report based on 116 cases. Am J Obstet Gynecol. 
2020 Apr 23;

10.1016/j.ajog.2020.04.014 [Link]

27. Hantoushzadeh S, Shamshirsaz A, Aleyasin A, Seferovic M, Kazemi S, Arian $\mathrm{S}$, et al. Maternal Death Due to COVID19 Disease. Am J Obstet Gynecol. 2020 Apr 28; doi: 10.1016/j.ajog.2020.04.030 [Link]

28. Buosenso D, Costa S, Sanguinetti M, Cattani P, Posteraro B, Marchetti S, et al. Neonatal Late Onset Infection with Severe Acute Respiratory Syndrome Coronavirus 2. Am J Perinatol. 2020 May 2; doi: 10.1055/s-0040-1710541 [Link]

29. Breslin N, Baptiste C, Gyamfi C, Miller R, Martinez R, Bernstein $\mathrm{K}$, et al. Coronavirus disease 2019 infection among asymptomatic and symptomatic pregnant women: two weeks of confirmed presentations to an affiliated pair of New York City hospitals. Am J Obstet Gynecol MFM. 2020; 2(2): 100118. doi:

10.1016/j.ajogmf.2020.100118 [Link]

30. Schnettler W, Ahwell Y, Suhag A. Severe acute respiratory distress syndrome incoronavirus disease 2019-infected pregnancy: obstetric and intensive care considerations. Am J Obstet Gynecol MFM. 2020 Apr 10; doi: 10.1016/j.ajog.2020.0 4.005 [Link]

31. Alzamora M, Paredes $T$, Cáceres $D$, Webb C, Valdez L, La Rosa M. Severe COVID-19 during Pregnancy and Possible Vertical Transmission. Am J Perinatol. 2020 Apr 18; doi: 10.1055/s0040-1710050 [Link]

32. Londero A, Rosseti E, Pittini C, Cagnacci $A$, Driul L. Maternal age and the risk of adverse pregnancy outcomes: a retrospective cohort study. BMC Pregnancy Childbirth. 2019; 19(261): doi: 10.1186/s12884-019-2400-x [Link]

33. Qiao J. What are the risks of COVID-19 infection in pregnant women? The Lancet. 2020; 395(10226): 760-762. doi: 10.1016/S0140-6736(20)30365-2 [Link]

34. Li X, Xu S, Yu M, Wang K, Tao Y, Zhao Y, et al. Risk factors for severity and mortality in adult COVID-19 inpatients in Wuhan. J Allergy Clin Inmunol. 2020 Apr 12; doi: 10.1016/j.jaci.2020.04.006 [Link]
35. Matsushita K, Ding N, Kou M, Hu X, Chen $\mathrm{M}$, Gao $\mathrm{Y}$, et al. The relationship of COVID-19 severity with cardiovascular disease and its traditional risk factors: A systematic review and meta-analysis. BMC Yale. 2020 Apr 6; doi: 10.1101/2020.04.05.20054155 [Link]

36. Moriconi D, Masi S, Rebelos E, Virdis A, Manca M, De Marco S, et al. Obesity prolongs the hospital stay in patients affected by COVID-19, and may impact on SARS-COV-2 shedding. Obes Res Clin Pract. 2020 Jun 4; doi: 10.1016/j.orcp.2020.05.009 [Link]

37. Kalligeros $M$, Shehadeh $F$, Mylona E, Benitez G, Beckwith C, Chan P, et al. Association of Obesity With Disease Severity Among Patients With Coronavirus Disease 2019. Obesity (Silver Spring). 2020 Apr 20; doi: 10.1002/oby.22859 [Link]

38. Fu L, Wang B, Yuan T, Chen X, Ao Y, Fitzpatrick $T$, et al. Clinical characteristics of coronavirus disease 2019 (COVID-19) in China: A systematic review and meta-analysis. J Infect. 2020; 80(6): 656-665. doi 10.1016/j.jinf.2020.03.04 [Link]

39. Escobar G, Matta J, Taype W, Ayala R, Amado J. Características clínicoepidemiológicas de pacientes fallecidos por COVID-19, en un hospital nacional de Lima, Perú. Rev. Fac. Med. Hum. 2020; 20(2): 180-185. doi: 10.25176/RFMH.v20i2.2940 [Link]

40. Fang Z, Yi F, Wu K, Lai K, Sun X, Zhong $\mathrm{N}$, et al. Clinical Characteristics of Coronavirus Pneumonia 2019 (COVID19): An Updated Systematic Review. Eur PMC. 2020 Mar 09; doi: 10.1101/2020.03.07.20032573 [Link]

41. Ai T, Yang Z, Hou H, Zhan C, Chen C, Lv W, et al. Correlation of Chest CT and RTPCR Testing in Coronavirus Disease 2019 (COVID-19) in China: A Report of 1014 Cases. Radiology. 2020 Feb 26; doi: 10.1148/radiol.2020200642 [Link]

42. Sun Z, Zhang N, Li Y, Xu X. A systematic review of chest imaging findings in COVID-19. Quant Imaging Med Surg. 2020; 10(5): 1058-1079. doi: 10.21037/qims-20-564 [Link]
43. Chen J, Qi T, Liu L, Ling Y, Qian Z, Li T, et al. Clinical progression of patients with COVID-19 in Shanghai, China. J Infect. 2020; 80(5): e1-e6. doi: 10.1016/j.jinf.2020.03.004 [Link]

44. Lopes J, Sass N, Martins S Preeclampsia. Rev Bras Ginecol Obtet. 2017; 39(9): 496-512. doi: 10.1055/s0037-1604471 [Link]

45. American College of Obstetricians and Gynecologists (ACOG), Society for Maternal Fetal Medicine (SFTM). Safe prevention of the primary cesarean delivery. Am J Obstet Gynecol. 2014; 2010(3): 179-193. doi 10.1016/j.ajog.2014.01.026 [Link]

46. Putra M, Kesavan M, Brackney K, Hackney D, Roosa M. Forecasting the Impact of Coronavirus Disease During Delivery Hospitalization: An Aid for Resources Utilization. Am J Obstet Gynecol MFM. 2020 Apr 25; doi: 10.1016/j.ajogmf.2020.100127 [Link]

47. Mimouni F, Lakshminrusimha S, Pearlman S, Raju T, Gallagher P, Mendlovic J. Perinatal aspects on the covid-19 pandemic: a practical resource for perinatal-neonatal specialists. J Perinatol. 2020; 40(5): 820-826. doi: 10.1038/s41372-020-0665-6 [Link]

48. Rasmussen S, Jamieson D. Coronavirus Disease 2019 (COVID-19) and Pregnancy: Responding to a Rapidly Evolving Situation. Obstet Gynecol. 2020; 135(5): 999-1002. doi: 10.1097/AOG.0000000000003873 [Link] 